Katarzyna Chmielewska, Institute of Mathematics, Bydgoszcz Academy, pl. Weyssenhoffa 11, 85-072 Bydgoszcz, Poland.

e-mail: kasiach@wsp.bydgoszcz.pl

\title{
ON STRONG PRODUCT MEASURABILITY
}

\author{
Abstract \\ We consider a special condition implying the strong product mea- \\ surability of bounded functions with values in a Banach space which all \\ vertical sections are derivatives.
}

Let $\mathbb{R}, \mathbb{Z}$, and $\mathbb{N}$ denote the real line, the set of integers, and the set of positive integers, respectively. Let $(X, \mathcal{M})$ be a measurable space, let $(Y, \mathcal{N}, \mu)$ be a measure space, and let $Z$ be a Banach space. We define $\mu_{i}$ as the inner measure generated by $\mu$, i.e., $\mu_{i}(C)=\sup \{\mu(D): D \in \mathcal{N}, D \subset C\}$ for each $C \subset Y$. Let $\mathcal{I} \subset \mathcal{M}$ be a proper $\sigma$-ideal of subsets of $X$. We assume that condition (ccc) is fulfilled in $(X, \mathcal{M}, \mathcal{I})$, i.e., that each family of pairwise disjoint elements of $\mathcal{M} \backslash \mathcal{I}$ is at most countable.

We assume that we have a net structure $\mathcal{J}$ in $(Y, \mathcal{N}, \mu)$ (see [1]). Recall that a net in $Y$ is an at most countable collection of pairwise disjoint measurable sets of positive measure which cover $Y$. The individual sets in the collection are called cells. The family $\mathcal{J}=\bigcup_{n=1}^{\infty} \mathcal{J}_{n}$, where each $\mathcal{J}_{n}$ is a net, is called a net structure. For each $y \in Y$, there is a sequence $\left(J_{n}\right)$ such that $y \in J_{n} \in \mathcal{J}_{n}$ for each $n$. In this case we write $J_{n} \Rightarrow y$. Several examples of net structures can be found, e.g., in [5] or [6].

Example 1. For each $n \in \mathbb{N}$, the family $\mathcal{J}_{n}$ of all intervals of the form

$$
\left[\frac{i_{1}-1}{2^{n}}, \frac{i_{1}}{2^{n}}\right) \times \cdots \times\left[\frac{i_{m}-1}{2^{n}}, \frac{i_{m}}{2^{n}}\right),
$$

where $i_{1}, \ldots, i_{m} \in \mathbb{Z}$, is a net in $\mathbb{R}^{m}$.

A function $g: Y \rightarrow Z$ is called a derivative (with respect to the net $\mathcal{J}$ ) if $g$ is Bochner-integrable over each $J \in \mathcal{J}$ and

$$
\lim _{n \rightarrow \infty} \frac{\int_{J_{n}} g}{\mu\left(J_{n}\right)}=g(y)
$$

Key Words: derivative, Baire property, measurability, strong measurability

Mathematical Reviews subject classification: 26B05, 26B99, 26A15

Received by the editors February 11, 2000

* Supported by Bydgoszcz Academy grant (1999). 
whenever $J_{n} \Rightarrow y, y \in Y$.

Let $h: X \rightarrow Z$. We say that $h$ is measurable if $h^{-1}(U) \in \mathcal{M}$ for every open set $U \subset Z$. We say that $h$ is nearly simple if there exists a sequence of pairwise disjoint sets $A_{1}, A_{2}, \ldots \in \mathcal{M}$ and elements $\alpha_{1}, \alpha_{2}, \ldots \in Z$, such that $h=\alpha_{n}$ on $A_{n}$ for each $n$, and $X=\bigcup_{n=1}^{\infty} A_{n}$. We say that $h$ is strongly measurable if there exists a sequence of nearly simple functions $\left(h_{n}\right)$ and a set $A \in \mathcal{I}$ such that $h_{n} \rightarrow h$ on $X \backslash A$. We will see that if $h$ is a strongly measurable function, then there exists a sequence of nearly simple functions uniformly convergent to $h$ outside of some $A \in \mathcal{I}$ (Proposition 5).

First we will investigate the properties of strongly measurable functions. Since each nearly simple function is measurable and the pointwise limit of measurable functions is measurable, each strongly measurable function is measurable as well.

Lemma 1. If $X=\bigcup_{n \in \mathbb{N}} X_{n}$, each $X_{n}$ is measurable, and $h \uparrow X_{n}$ is nearly simple (strongly measurable) for each $n$, then $h$ is nearly simple (respectively strongly measurable).

Proof. For each $n$ let $A_{n 1}, A_{n 2}, \ldots \in \mathcal{M}$ and $\alpha_{n 1}, \alpha_{n 2}, \cdots \in Z$ be such that $h=\alpha_{n k}$ on $A_{n k}$ for each $k$, and $X_{n}=\bigcup_{k=1}^{\infty} A_{n k}$. Arrange all sets of the form $A_{n k} \backslash \bigcup_{i<n} X_{i}$ in a sequence, $\left(B_{m}\right)$, and let $\beta_{m} \in Z$ be such that $h=\beta_{m}$ on $B_{m}$. Clearly each $B_{m}$ is measurable and $X=\bigcup_{m=1}^{\infty} B_{m}$.

Now suppose that each $h \uparrow X_{n}$ is strongly measurable. For each $n$ choose a sequence of nearly simple functions, $\left(h_{n k}\right)$, and a set $A_{n} \in \mathcal{I}$ such that $h_{n k} \rightarrow h$ on $X_{n} \backslash A_{n}$. For each $k$ define $g_{k}=h_{n k}$ on $X_{n} \backslash \bigcup_{i<n} X_{i}$. Then each $g_{k}$ is nearly simple and evidently $g_{k} \rightarrow h$ on $X \backslash \bigcup_{n=1}^{\infty} A_{n}$.

Lemma 2. Let $h, h_{1}, h_{2}, \ldots: X \rightarrow Z$. If each of the sets $h_{1}(X), h_{2}(X), \ldots$ is a separable subspace of $Z$ and $h_{n} \rightarrow h$, then $h(X)$ is a separable subspace of $Z$.

Proof. Since $Z_{0}=\operatorname{cl}\left(\bigcup_{n=1}^{\infty} h_{n}(X)\right)$ is a separable subspace of $Z$, it suffices to prove that $h(X) \subset Z_{0}$. Let $z \in h(X)$. There exists an $x \in X$ such that $h(x)=z$. Since $h_{n}(x) \rightarrow z$, we get $z \in \operatorname{cl}\left\{h_{n}(x): n \in \mathbb{N}\right\} \subset Z_{0}$.

Clearly if $h$ is a nearly simple function, then $h(X)$ is a separable subspace of $Z$. So by Lemma 2, we obtain the following corollary.

Corollary 3. Let $h: X \rightarrow Z$. If $h$ is a strongly measurable function, then there exists a set $A \in \mathcal{I}$ such that $h(X \backslash A)$ is a separable subspace of $Z$.

Proof. Let $\left(h_{n}\right)$ be a sequence of nearly simple functions pointwise convergent to $h$ on $X \backslash A$, where $A \in \mathcal{I}$. Then by Lemma $2, h(X \backslash A)$ is a separable subspace of $Z$. 
Lemma 4. Let $h: X \rightarrow Z$. If $h$ is a measurable function and $h(X)$ is a separable subspace of $Z$, then $h$ is strongly measurable. Moreover there is a sequence of nearly simple functions uniformly convergent to $h$ on $X$.

Proof. Let $\varepsilon>0$. Let $\left\{p_{n}: n<N\right\}$ be an at most countable dense subset of $h(X)$. For each $n<N$ define

$$
A_{n}=h^{-1}\left(B\left(p_{n}, \varepsilon\right)\right) \backslash \bigcup_{k<n} h^{-1}\left(B\left(p_{k}, \varepsilon\right)\right),
$$

where $B\left(p_{n}, \varepsilon\right)=\left\{z \in Z:\left\|z-p_{n}\right\|<\varepsilon\right\}$. Define $\varphi(x)=p_{n}$ if $x \in A_{n}$, $n<N$. Clearly $\varphi$ is a nearly simple function and $\|\varphi(x)-h(x)\|<\varepsilon$ for each $x \in X$.

Proposition 5. If $h$ is a pointwise limit of a sequence of strongly measurable functions, then $h$ is strongly measurable. Moreover there exists a sequence of nearly simple functions $\left(\varphi_{n}\right)$ and a set $A \in \mathcal{I}$ such that $\left(\varphi_{n}\right)$ is uniformly convergent to $h$ on $X \backslash A$.

Proof. Let $\left(h_{n}\right)$ be a sequence of strongly measurable functions pointwise convergent to $h$. For each $n$ let $A_{n} \in \mathcal{I}$ be such that $h_{n}\left(X \backslash A_{n}\right)$ is a separable subspace of $Z$. (See Corollary 3.) Then $A=\bigcup_{n \in \mathbb{N}} A_{n} \in \mathcal{I}$ and by Lemma 2, $h(X \backslash A)$ is a separable subspace of $Z$. Now our assertion follows from Lemma 4.

Example 2. Assume $Z$ is nonseparable and let $h:\left(Z, 2^{Z},\{\emptyset\}\right) \rightarrow Z$ be an arbitrary function. Then evidently $h$ is measurable, but by Corollary 3 and Lemma $4, h$ is strongly measurable if and only if $h(Z)$ is separable.

We will use the following generalization of Davies' Lemma [3].

Proposition 6. Assume that a function $h: X \rightarrow Z$ fulfills the following condition:

for each $\varepsilon>0$ and each $A \in \mathcal{M} \backslash \mathcal{I}$, there is a $B \in \mathcal{M} \backslash \mathcal{I}$ with $B \subset A$ such that $\operatorname{osc}_{B} h<\varepsilon$,

where $\operatorname{osc}_{B} h=\sup \{\|h(x)-h(t)\|: x, t \in B\}$. Then $h$ is strongly measurable.

Proof. Fix an $\varepsilon>0$. It suffices to show that there are a set $K \in \mathcal{I}$ and a nearly simple function $\varphi$ such that $\|\varphi(x)-h(x)\|<\varepsilon$ for each $x \in X \backslash A$. Let $\Omega$ denote the first uncountable ordinal. First we will define by transfinite induction a sequence $\left\{A_{\alpha}: \alpha<\Omega\right\}$, composed of pairwise disjoint elements of $\mathcal{M}$, such that for each $\alpha<\Omega$, if $A_{\alpha} \in \mathcal{I}$, then $A_{\alpha}=\emptyset$.

Let $\alpha<\Omega$ and suppose we have already defined the set $A_{\beta}$ for each $\beta<\alpha$. Set $K=X \backslash \bigcup_{\beta<\alpha} A_{\beta}$. If $K \in \mathcal{I}$, then we define $A_{\alpha}=\emptyset$. In the opposite 
case observe that $K \in \mathcal{M}$. (Recall that $\alpha<\Omega$.) Use condition (1) to find a set $A_{\alpha} \in \mathcal{M} \backslash \mathcal{I}$ such that $A_{\alpha} \subset K$ and $\operatorname{osc}_{A_{\alpha}} f<\varepsilon$.

By (ccc) there is a $\xi<\Omega$ such that $A_{\alpha}=\emptyset$ for each $\alpha \geq \xi$. We may assume that $A_{\alpha} \neq \emptyset$ for each $\alpha<\xi$. Define $K=X \backslash \bigcup_{\alpha<\xi} A_{\alpha}$. For each $\alpha<\xi$ choose an $x_{\alpha} \in A_{\alpha}$. Define $\varphi(x)=f\left(x_{\alpha}\right)$ if $x \in A_{\alpha}$ for some $\alpha<\xi$, and $\varphi(x)=f\left(x_{0}\right)$ if $x \in K$. Since $A_{\xi}=\emptyset$, we have $K \in \mathcal{I}$. So, $\varphi$ is a nearly simple function. (We use the fact that $\xi<\Omega$.) Clearly $\|\varphi(x)-h(x)\|<\varepsilon$ for each $x \in X \backslash A$.

Let $f: X \times Y \rightarrow Z$. In the space $X \times Y$, we consider the product $\sigma$-field $\mathcal{M} \times \mathcal{N}$ (i.e., the $\sigma$-field generated by the family of all sets of the form $M \times N$, where $M \in \mathcal{M}$ and $N \in \mathcal{N}$ ), and the $\sigma$-ideal $\{A \times B: A \in \mathcal{I}, B \subset Y\}$. So, $f$ is nearly simple if there are elements $\alpha_{1}, \alpha_{2}, \ldots \in Z$ and a sequence of pairwise disjoint sets $A_{1}, A_{2}, \ldots \in \mathcal{M} \times \mathcal{N}$, such that $f=\alpha_{n}$ on $A_{n}$ for each $n$, and $X \times Y=\bigcup_{n=1}^{\infty} A_{n} ; f$ is strongly measurable if there exists a sequence of nearly simple functions $\left(f_{n}\right)$ and a set $A \in \mathcal{I}$ such that $f_{n} \rightarrow f$ on $(X \times Y) \backslash(A \times Y)$. For each $x \in X$, we define the section $f_{x}: Y \rightarrow Z$ by $f_{x}(y)=f(x, y)$. Analogously, for each $y \in Y$, the section $f^{y}: X \rightarrow Z$ is defined by $f^{y}(x)=f(x, y)$.

Theorem 7. Assume that a bounded function $f: X \times Y \rightarrow Z$, which all sections $f_{x}$ are derivatives, satisfies the following condition:

for every $\varepsilon>0$ and every set $A \in \mathcal{M} \backslash \mathcal{I}$, there exist a $B \in \mathcal{M} \backslash \mathcal{I}$ with $B \subset A$ and $a C \subset Y$ such that $\mu_{i}(Y \backslash C)<\varepsilon$ and $\operatorname{osc}_{B} f^{y}<\varepsilon$ for every $y \in C$.

Then $f$ is strongly measurable.

Proof. Let $M$ be such that $\|f(x, y)\|<M / 4$ for all $(x, y) \in X \times Y$. Fix a $J \in \mathcal{J}$, and put $h_{J}(x)=\int_{J} f_{x}$. We will prove that $h_{J}$ is strongly measurable. We will use Proposition 6. Fix an $\varepsilon>0$ and an $A \in \mathcal{M} \backslash I$. By (2), there are a $B \in \mathcal{M} \backslash \mathcal{I}$ with $B \subset A$ and a $C \subset Y$ such that

$$
\mu_{i}(Y \backslash C)<\varepsilon / M
$$

and

$$
\operatorname{osc}_{B} f^{y}<\frac{\varepsilon}{4 \mu(J)} \quad \text { for every } y \in C .
$$

Fix points $u, v \in B$. Define

$$
D=\left\{y \in J:\|f(u, y)-f(v, y)\|<\frac{\varepsilon}{4 \mu(J)}\right\} .
$$


Since the sections $f_{u}$ and $f_{v}$ are derivatives, the set $D$ is $\mathcal{N}$-measurable. By (4), we get $D \supset C$. So by $(3), \mu(J \backslash D)<\varepsilon / M$. Consequently,

$$
\begin{aligned}
\left\|h_{J}(u)-h_{J}(v)\right\| & =\left\|\int_{J} f_{u}-\int_{J} f_{v}\right\| \leq \int_{D}\left\|f_{u}-f_{v}\right\|+\int_{J \backslash D}\left\|f_{u}-f_{v}\right\| \\
& \leq \frac{\varepsilon}{4 \mu(J)} \cdot \mu(J)+\frac{\varepsilon}{M} \cdot \frac{M}{2}=\frac{3 \varepsilon}{4}
\end{aligned}
$$

and $\operatorname{osc}_{B} h_{J}<\varepsilon$. By Proposition 6 , we conclude that $h_{J}$ is strongly measurable.

Now we will repeat the Lipiński's argument [4] to show the strong measurability of $f$. For each $n \in \mathbb{N}$ define

$$
f_{n}(x, y)=h_{J}(x) / \mu(J)
$$

if $y \in J \in \mathcal{J}_{n}$. By Lemma 1, each function $f_{n}$ is strongly measurable. We will show that $f_{n} \rightarrow f$, whence by Proposition 5 , we will obtain the assertion of the theorem.

Fix a point $(x, y) \in X \times Y$. Let $J_{n} \Rightarrow y$. Using the fact that $f_{x}$ is a derivative, we obtain

$$
f(x, y)=f_{x}(y)=\lim _{n \rightarrow \infty} \int_{J_{n}} f_{x} / \mu\left(J_{n}\right)=\lim _{n \rightarrow \infty} h_{J_{n}}(x) / \mu\left(J_{n}\right)=\lim _{n \rightarrow \infty} f_{n}(x, y) .
$$

This completes the proof.

Remark 1. By Remark 2 of [2], if each set $A \subset \mathbb{R}$ of cardinality less than that of continuum has Lebesgue measure zero, then there is a Lebesgue measurable function $f: \mathbb{R}^{2} \rightarrow[0,1]$ which all sections $f_{x}$ and $f^{y}$ are derivatives and which does not satisfy condition (2). So, condition (2) is not necessary for the measurability of a bounded function $f$ which all sections $f_{x}$ and $f^{y}$ are derivatives.

The following theorem is proved in [2].

Theorem 8. Assume that a locally bounded function $f: \mathbb{R}^{2} \rightarrow \mathbb{R}$ which all sections $f_{x}, x \in \mathbb{R}$, are derivatives, satisfies the following condition:

for every $\varepsilon>0$ and every open interval $I$, there exist an $x \in I$ and a linear set $D$ such that $\mu_{i}(\mathbb{R} \backslash D)<\varepsilon$ and for every $y \in D$ there is an open interval $I_{y} \ni x$ such that $\operatorname{osc}_{I_{y}} f^{y}<\varepsilon$.

Then $f$ has the Baire property.

Observe that in general condition (5) does not imply condition (2). 
Example 3. Define $f: \mathbb{R}^{2} \rightarrow \mathbb{R}$ by $f(x, y)=x y$. Then $f$ is continuous, so $f$ fulfills condition (5). Notice that if $a<b$ and $\left|f^{y}(a)-f^{y}(b)\right|=|y(b-a)|<1$, then $|y|<(b-a)^{-1}$. So, for each set $B \subset \mathbb{R}$ with cardinality greater than 1 the set $\left\{y \in \mathbb{R}: \operatorname{osc}_{B} f^{y}<1\right\}$ is bounded. Consequently, condition (2) (where $\mathcal{M}$ denotes the $\sigma$-field of all sets with the Baire property and $\mathcal{I}$ is the $\sigma$-ideal of meager sets) fails.

Despite of that we will show that Theorem 8 is actually a particular case of Theorem 7 .

Theorem 9. Assume that $f$ satisfies the assumptions of Theorem 8. Then for each $m \in \mathbb{N}$, the restricted function $f \uparrow[-m, m]^{2}$ fulfills condition (2). So, by Theorem 7 and Lemma 1, $f$ has the Baire property.

Proof. Fix an $\varepsilon>0$ and an $A \subset \mathcal{M} \backslash \mathcal{I}$. There is an open interval $I$ such that $I \backslash A \in \mathcal{I}$. Use condition (5) to find an $x \in I$ and a $D \subset[-m, m]$ such that $\mu_{i}([-m, m] \backslash D)<\varepsilon$ and for every $y \in D$ there is an open interval $I_{y} \ni x$ such that $\operatorname{osc}_{I_{y}} f^{y}<\varepsilon$. For each $n \in \mathbb{N}$ define

$$
D_{n}=\left\{y \in D: I_{y} \supset\left(x-n^{-1}, x+n^{-1}\right)\right\} .
$$

Then $D=\bigcup_{n \in \mathbb{N}} D_{n}$. Since $\left(D_{n}\right)$ is an ascending sequence of sets,

$$
\mu_{i}\left([-m, m] \backslash D_{n}\right)<\varepsilon
$$

for some $n \in \mathbb{N}$. Put $B=\left(x-n^{-1}, x+n^{-1}\right) \cap I \cap A$ and $C=D_{n}$. Evidently $B \subset A, B \in \mathcal{M} \backslash \mathcal{I}$, and $\mu_{i}([-m, m] \backslash C)<\varepsilon$. Moreover

$$
\operatorname{osc}_{B} f^{y} \leq \operatorname{osc}_{I_{y}} f^{y}<\varepsilon
$$

for every $y \in C$.

Example 4. Define $f: \mathbb{R}^{2} \rightarrow \mathbb{R}$ by

$$
f(x, y)= \begin{cases}1 & \text { if } x \in \mathbb{Q} \\ 0 & \text { otherwise }\end{cases}
$$

Then function $f$ has the Baire property. It fulfills condition (2) if we define $\mathcal{M}$ and $\mathcal{I}$ as in Example 3. Meanwhile one can readily verify that for each $y \in \mathbb{R}$ and each open interval $J$ we have $\operatorname{osc}_{J} f^{y}=1$. So, condition (5) fails. 


\section{References}

[1] A. M. Bruckner, Differentiation of integrals, p. II, Amer. Math. Monthly, 78(9), 1971.

[2] K. Chmielewska, Z. Grande, On the measurability and the Baire property of functions of two variables which all vertical sections are derivatives, to appear in Tatra Mt. Math. Publ.

[3] R. O. Davies, Separate approximate continuity implies measurability, Proc. Camb. Phil. Soc., 73(1973), 461-465.

[4] J. S. Lipiński, On measurability of functions of two variables, Bull. Acad. Polon. Sci., Sér. Sci. Math. Astronom. Phys., 20(1972), 131-135.

[5] L. Mišík, Über den Mittelwertsatz für additive Zellenfunktionen, Matematicko-Fizykalny Casopis SAV, 15(4), (1963), 260-274.

[6] C. Pauc, Dérivées et Intégrales: Fonctions de cellule, Conférence faite au Centre Mathématique International de Varenna, 15-25 Aug. 1954, pub. Mathematical Institute, Rome. 
